REVIEW

\title{
Anti-RhD immunoglobulin in the treatment of immune thrombocytopenia
}

\author{
Eric Cheung \\ Howard A Liebman
}

Jane Anne Nohl Division of Hematology and Center for the Study of Blood Diseases, University of Southern California-Keck School of Medicine, Los Angeles, CA, USA
Correspondence: Howard A Liebman Norris Cancer Hospital, Rm 3466, I44I Eastlake Ave, Los Angeles, CA 90033, USA $\mathrm{Tel}+\mathrm{I} 3238653950$

Fax + I 3238650060

Email liebman@usc.edu

\begin{abstract}
Immune thrombocytopenia (ITP) is an acquired bleeding autoimmune disorder characterized by a markedly decreased blood platelet count. The disorder is variable, frequently having an acute onset of limited duration in children and a more chronic course in adults. A number of therapeutic agents have demonstrated efficacy in increasing the platelet counts in both children and adults. Anti-RhD immunoglobulin (anti-D) is one such agent, and has been successfully used in the setting of both acute and chronic immune thrombocytopenia. In this report we review the use of anti-D in the management of ITP. While the FDA-approved dose of $50 \mathrm{mg} / \mathrm{kg}$ has documented efficacy in increasing platelet counts in approximately $80 \%$ of children and $70 \%$ of adults, a higher dose of $75 \mu \mathrm{g} / \mathrm{kg}$ has been shown to result in a more rapid increase in platelet count without a greater reduction in hemoglobin. Anti-D is generally ineffective in patients who have failed splenectomy. Anti-RhD therapy has been shown capable of delaying splenectomy in adult patients, but does not significantly increase the total number of patients in whom the procedure can be avoided. Anti-D therapy appears to inhibit macrophage phagocytosis by a combination of both FcR blockade and inflammatory cytokine inhibition of platelet phagocytosis within the spleen. Anti-RhD treatment is associated with mild to moderate infusion toxicities. Rare life-threatening toxicities such as hemoglobinuria, acute renal failure and disseminated intravascular coagulation have been reported. Recommendations have been proposed to reduce the risk of these complications. Anti-D immunoglobulin can be an effective option for rapidly increasing platelet counts in patients with symptomatic ITP.
\end{abstract}

Keywords: immune thrombocytopenia, RhD immunoglobulin

Immune thrombocytopenia (ITP) is an autoimmune disorder characterized by a decreased number of platelets that often results in mucocutaneous bleeding. ${ }^{1}$ ITP remains a diagnosis of exclusion. Although multiple associations have been recognized (secondary ITP), the etiology of idiopathic or primary ITP remains uncertain. ${ }^{1}$ ITP in adults typically has a remitting-relapsing course; while ITP in children typically occurs immediately after an infectious illness and most often resolves spontaneously, without further relapses. ${ }^{1,2}$ Secondary ITP has been associated with autoimmune disorders such as systemic lupus erythematosus, antiphospholipid antibody syndrome, or thyroid abnormalities, infections with human immunodeficiency virus, hepatitis $\mathrm{C}$, or Helicobacter pylori, and certain hematologic malignancies. ${ }^{3}$

Multiple mechanisms have been described in ITP, highlighting the heterogeneous nature of this disease. ${ }^{4}$ Anti-platelet auto-antibodies, directed against platelet membrane glycoproteins have been identified. ${ }^{4}$ Platelets coated with IgG auto-antibodies undergo rapid clearance through Fc $\gamma$ receptors on tissue macrophages. ${ }^{4}$ Auto-antibodies may also induce megakaryocytic apoptosis resulting in defective platelet production. ${ }^{4}$ Treatment of ITP targets different aspects of antibody-mediated platelet clearance, autoantibody production and/or immune-mediated defective platelet production. ${ }^{5}$ 
Common first-line treatments of ITP are intended to inhibit autoantibody-mediated platelet clearance. These would include the use of corticosteroids (such as prednisone, dexamethasone), intravenous immunoglobulin (IVIg) and anti-RhD immunoglobulin (anti-D). ${ }^{5}$ Other therapies may target autoantibody production as well as platelet clearance and can include splenectomy, danazol, azathioprine, cyclosphosphamide and anti-CD20 therapy with rituxamab. ${ }^{5}$ Recently, romiplostim, a thrombopoietin receptor agonist, has been approved for the treatment of refractory ITP. ${ }^{6}$ In this review we will focus on the use of anti-D immunoglobulin in the treatment of ITP.

\section{Anti-RhD immunoglobulin}

Anti-RhD immunoglobulin is composed of immunoglobulin $\mathrm{G}(\mathrm{IgG})$ prepared from the plasma of repeatedly immunized human RhD-negative donors. Anti-D IgG is manufactured from the plasma of donors and contains $>90 \%$ polyclonal immunoglobulin G anti-D. ${ }^{7}$ The concentration of IgG1, IgG2, and IgG3 are comparable to those of normal serum while the levels of IgG4 are negligible. ${ }^{7,8}$ There are also minimal concentrations of antibodies against other red blood cell antigens.

Anti-RhD was originally prepared to prevent hemolytic disease in newborns. ${ }^{9}$ Since 1983 , it has been used to treat patients with immune thrombocytopenia. ${ }^{9,10}$ In March of 1995, anti-D immunoglobulin was licensed by the United States Food and Drug Administration for the treatment of immune thrombocytopenic purpura in non-splenectomized children with acute ITP who were RhD-positive and children and adults with ITP secondary to human immunodeficiency infection. ${ }^{8}$

\section{History of clinical use}

In 1984, Salama et al published the first results on the use of intravenous anti-D in 10 Rh-positive ITP patients. ${ }^{9,10}$ The patients had chronic ITP with the duration of their disease ranging from 1 to 21 years. Nine patients were female, of whom 4 had previous splenectomy. They received an initial intravenous dose of 200 to $1000 \mu \mathrm{g}$ of anti-D immunoglobulin for a total dose of 300 to $3600 \mu \mathrm{g}$. On average, the platelets increased by $80 \%$. The platelet counts of splenectomized patients increased by $16 \times 10^{9} / \mathrm{L}$ while the average increase in non-splenectomized patients was $60 \times 10^{9} / \mathrm{L}$. The increased platelet count lasted from 7 to over 150 days. A transient but mild hemolysis was observed in 7 of 10 patients.

\section{Anti-D treatment in children}

In 1986, Panzer et al compared the efficacy of $\operatorname{IVIg}(0.4 \mathrm{~g} / \mathrm{kg} \times$ 5 days) with anti-D (11-20 $\mu \mathrm{g} / \mathrm{kg} \times 5$ days $)$ in 5 patients with ITP, 2 of whom were children. ${ }^{11}$ The one child with acute
ITP who received anti-D had a platelet increase of $11 \times 10^{9} / \mathrm{L}$ while the child with chronic ITP had a platelet increase of $22 \times 10^{9} / \mathrm{L}$. Neither child had a significant decrease in their hemoglobin levels. In the same year, a collaborative study of $15 \mathrm{Rh}$-positive children with ITP were treated with low-dose anti-D (28-50 $\mu \mathrm{g} / \mathrm{kg}$ intravenously) was reported by Becker and colleagues. ${ }^{12}$ Ten patients had chronic ITP (duration of 6-47 months), while 5 had acute ITP. All patients had resolution of their bleeding and increased in platelet counts without a significant decrease in their hemoglobin or other adverse effects exclusive of minor transfusion related symptoms. ${ }^{12}$ In the children with chronic ITP the duration of the platelet count increase ranged from 10 to over 360 days.

In 1991, Bussel et al treated 46 patients with ITP, of which 20 were children. ${ }^{13}$ Anti-D was given at a treatment dose of $50 \mu \mathrm{g} / \mathrm{kg} .{ }^{13}$ Of the 20 children, 14 had ITP for greater than 6 months and 3 children were HIV-positive. Platelet increases were greater in children than adults (median $92 \times 10^{9} / \mathrm{L}$ versus $\left.26 \times 10^{9} / \mathrm{L}\right)$. Of the 14 children with chronic disease, 2 achieved remission and 3 became stable after 5 treatments.

Andrew et al performed a multicenter cohort study of escalating doses of intravenous anti-D in children with chronic ITP (greater than 6 months' duration). ${ }^{14}$ Twenty-five $\mathrm{Rh}$-positive children received doses of anti-D that increased from $25 \mu \mathrm{g} / \mathrm{kg}$ upwards to $55 \mu \mathrm{g} / \mathrm{kg}$. By day 7 of the protocol, 23 patients had responded to therapy, 18 of whom had platelet counts greater than $150,000 / \mu \mathrm{L}$. The median duration of response was 5 weeks (range 1 to 24 weeks). Of the 23 responding patients, 21 were retreated and 18 responded to the second course of therapy. By the completion of the study, 16 patients continued to have excellent responses to intermittent anti-D therapy.

A randomized study of 146 children with acute ITP with platelet counts below $20,000 / \mu \mathrm{L}$ compared high dose IVIg $(1 \mathrm{~g} / \mathrm{kg} \times 2$ days or $0.8 \mathrm{~g} / \mathrm{kg} \times 1)$, intravenous anti-D $(25 \mu \mathrm{g} / \mathrm{kg} \times 2$ days), or oral prednisone ( $4 \mathrm{mg} / \mathrm{kg} /$ day with tapering and discontinuation by day 21). ${ }^{15}$ The increase in platelets was significantly faster for patients receiving IVIg compared to anti-D. The hemoglobin fell to less than $10 \mathrm{~g} / \mathrm{dL}$ in $24 \%$ of the patients receiving anti-D. The authors were unable to recommend anti-D as initial therapy for children with acute ITP with platelet counts lower than $20,000 / \mu \mathrm{L}$. Borgna-Pignatti et al published a series on 7 pediatric patients with chronic ITP who were treated with intramuscular anti-D for 5 times on an alternate-day basis. ${ }^{16}$ Five patients responded to therapy, 2 of whom went into long-term remission. No patient experienced significant anemia despite evidence of low-grade hemolysis. 
In 1997, Scaradavou et al published the largest study of anti-D use in ITP patients. ${ }^{17}$ Two hundred seventytwo patients were treated, including 124 children and 137 adult chronic ITP patients, of which 105 patients (children and adults) were HIV-positive. An additional 11 post-splenectomy patients were also treated. The patients received 25 or $50 \mu \mathrm{g} / \mathrm{kg} /$ dose of intravenous anti-D, with the post-splenectomy patients receiving 100-200 $\mu \mathrm{g} / \mathrm{kg} / \mathrm{dose}$. The best treatment responses were observed in the HIVnegative children. HIV-negative pediatric patients had a significantly higher mean platelet increase $\left(119,000 \times 10^{9} / \mathrm{L}\right)$ when compared to the HIV-infected children $\left(65 \times 10^{9} / \mathrm{L}\right.$; $\mathrm{p}=0.005)$. Also the number of responding HIV-negative children $(83 \%)$ was significantly greater than that observed in the HIV-negative adults $(69 \% ; \mathrm{p}=0.05)$. Anti-D therapy at doses given was again shown to be ineffective in patients who failed splectectomy. The inability of the drug to work in this patient population may reflect of different pathophysiology of the disease in splenectomy failures such a antibody mediated defective megakaryopoiesis. ${ }^{18}$

\section{Anti-D treatment in adults}

Immune thrombocytopenia in adults is most often chronic in nature. Many patients, requiring multiple therapeutic interventions. Anti-D treatment, along with intravenous Ig, remains one of the few non-immunosuppressive therapies capable of acutely increasing platelet counts in patients with severe thrombocytopenia.

Two years following his 1984 publication, Salama reported a study of anti-D treatment in 17 adults patients with ITP (15 chronic ITP patients and 2 acute ITP). ${ }^{19}$ The patients were given intravenous and/or intramuscular injections of anti-D. An average increase in the platelet count of $50 \times 10^{9} / \mathrm{L}$ was achieved in 13 of the $\mathrm{Rh}$-positive patients and none of the Rh-negative patients. ${ }^{19}$ A long-term response of greater than 5 months was achieved in 5 patients. Overt hemolysis was observed in one patient. ${ }^{19}$ Rossi and colleagues treated 13 adult HIV-positive ITP patients with varying doses of anti-D. ${ }^{20} \mathrm{~A}$ mean platelet count increase to greater than $100 \times 10^{9} / \mathrm{L}$ was observed and response was associated with a decrease in the patients' hemoglobin ranging between 1 and $1.7 \mathrm{~g} / \mathrm{dL}$.

Boughton et al reported a study of 13 Rh-positive adult patients with chronic ITP who received varying intravenous doses of anti-D. ${ }^{21}$ Significant increases in platelet counts were seen in all patients, especially those who received $12,500 \mathrm{IU}$ of anti-D. No adverse effects were observed in the patient groups. In the same year, Oksenhendler examined the effects of intravenous anti-D $(12-25 \mu \mathrm{g} / \mathrm{kg} \times 2$ days $)$ on 17 adult HIV-positive ITP patients with platelet counts less than $20 \times 10^{9} / \mathrm{L}^{22}$ Nine patients $(53 \%)$ had a significant increase in their platelet counts to greater than $50 \times 10^{9} / \mathrm{L}$.

In 1992, Gringeri et al performed a prospective study in 51 ITP patients, 24 of whom were HIV-positive. ${ }^{23}$ Patients who responded to intravenous anti-D $(3 \mu \mathrm{g} / \mathrm{kg} \times 3$ days $)$ received intramuscular injections of anti-D $(6 \mu \mathrm{g} / \mathrm{kg} / \mathrm{week})$. Twenty of the 24 HIV-positive ITP patients had a platelet response as opposed to 14 of the 27 idiopathic chronic ITP patients. In the same year, Rodeghiero et al published the results of 49 adult patients treated with high-dose IVIg or anti-D. ${ }^{24}$ Response and remission rates did not vary significantly among the two groups. ${ }^{24}$

As mentioned earlier, the largest study to date was published in 1997 by Scaradavou et al. ${ }^{17}$ In this study, 137 adult patients, including both HIV negative and positive patients, were treated with anti-D. In contrast to the results observed in the pediatric patients, HIV infection of adult ITP patients did not influence their response to anti-D. Baseline hemoglobin significantly affected platelet response to anti-D in adults with the mean platelet increase $\left(51 \times 10^{9} / \mathrm{L}\right.$ versus $\left.21 \times 10^{9} / \mathrm{L}\right)$ and response rate $(72 \%$ versus $42 \%)$ significantly higher in patients with hemoglobin levels $\geq 12 \mathrm{~g} / \mathrm{dL}$.

\section{Dosing: 50 mg/kg versus $75 \mu \mathrm{g} / \mathrm{kg}$}

The approved dosing regimen for anti-D therapy in the United States is $50 \mu \mathrm{g} / \mathrm{kg}$. However, significant questions remain as to whether higher doses may be safe and more efficacious. Newman and colleagues, reported on a randomized study comparing $75 \mu \mathrm{g} / \mathrm{kg}$ anti-D to the approved dose of $50 \mu \mathrm{g} / \mathrm{kg} .{ }^{25}$ Previous studies reported that a maximal platelet response to $50 \mu \mathrm{g} / \mathrm{kg} / \mathrm{d}$ occurred 72 hours after intravenous infusion. Twenty-seven adult, HIV-negative, non-splenectomized patients with initial platelet counts less than $30 \times 10^{9} / \mathrm{L}$ were studied. The $75 \mu \mathrm{g} / \mathrm{kg} /$ day dose resulted in greater median platelet increases on days 1 and 7, when compared with the $50 \mu \mathrm{g} / \mathrm{kg}$ dose, without a significantly greater decrease in hemoglobin. ${ }^{25}$

\section{Anti-D maintenance treatment to avoid or delay splenectomy:}

Cooper et al examined whether repeated infusions of anti-D could allow adults ITP patients, who had failed initial corticosteroid therapy, avoid splenectomy. ${ }^{26}$ Twenty-eight adult patients with platelet counts of less than $30 \times 10^{9} / \mathrm{L}$ were repeatedly treated with anti-D whenever their platelet count decreased to less than $30 \times 10^{9} / \mathrm{L}$. A response was defined as 
an incremental platelet increase greater than $20 \times 10^{9} / \mathrm{L}$ with an absolute value greater than $30 \times 10^{9} / \mathrm{L}$ within 7 days of therapy. Twenty-six (93\%) patients responded to initial anti-D treatment and 19 patients $(68 \%)$ responded to repeated treatments. After a median follow-up of 26 months, 19 patients avoided splenectomy; including 12 (43\%) patients who were off treatment for more than 6 months without requiring a splenectomy. At study entry, a platelet count greater than $14 \times 10^{9} / \mathrm{L}$ was a primary predictor for patients more likely to successfully discontinue treatment with anti-D. ${ }^{26}$

In a second randomized study to evaluate the use of anti$\mathrm{D}$ as an alternative to corticosteroid therapy in patients who wish to avoid or delay splenectomy, 70 patients were randomized to either anti-D or corticosteroid therapy. A splenectomy was to be performed if a patient failed to respond to 3 consecutive anti-D treatments given within 10 days. ${ }^{27}$ Patients treated with repeat doses of anti-D were able to avoid splenectomy for a median duration of 112 days as opposed to 36 days in the corticosteroid treated group and required fewer days of corticosteroid therapy. However, splenectomy was performed prematurely against study protocol in 11 of the 14 patients randomized to anti-D treatment and there was no statistical difference in the total number of patients in either treatment arm who subsequently had a splenectomy. ${ }^{27}$

\section{Mechanisms}

Multiple mechanisms whereby anti-D ameliorates thrombocytopenia have been proposed, including mononuclear phagocytic system (MPS) blockade, and cytokine modulation. ${ }^{28-30}$

The largest body of evidence exists for the competitive inhibition and blockade of the mononuclear phagocytic system by sensitized red blood cells within the spleen. Evidence to support this as a major mechanism of the therapy include multiple studies which show that anti-D is ineffective in $\mathrm{RhD}$ antigen-negative patients and relatively ineffective at standard doses in patients who have had a splenectomy. In vitro studies of cytokine expression from human monocytes and granulocytes exposed to anti-D coated red blood cells have demonstrated enhanced secretion of interleukin 1 receptor antagonist resulting in down-regulation of Fc $\gamma \mathrm{R}$ mediated phagocytosis. ${ }^{28}$ Murine models of ITP have demonstrated that RBC-specific antibodies can increase platelet counts by down-regulating Fc $\gamma$ RIIIa on splenic macrophage in a manner dissimilar to IVIg. ${ }^{29}$

A study evaluating the cytokine responses to infusions of IVIg and anti-D in patients with ITP demonstrated significant increases in interleukin 6 and 10, monocyte chemo-attractant protein-1, and tumor necrosis factor $\alpha$ within 2 hours of infusion of anti-D. ${ }^{30}$ Higher levels of Il-10 correlated with the greatest platelet increases at 24 hours. Patients with Fc $\gamma$ IIa$131 \mathrm{HH}$ genotype had higher cytokine responses and patients with Fc $\gamma$ IIIa-158VF had higher platelet counts by day $7 .{ }^{30}$ It would appear from these studies that anti-D therapy induces an initial inflammatory cytokine response mediated through the Fc $\gamma$ IIa receptor leading to a secondary suppression of FcyRIIIa mediated platelet phagocytosis. This is significantly different from the anti-inflammatory effect of IVIG in which Fc $\gamma I I I a$ and Fc $\gamma I I a$ receptors are down-regulated by interaction of IVIG with Fc $\gamma$ IIB receptors. ${ }^{28-31}$

\section{Toxicities associated with anti-D treatment}

In the original anti-D IGIV clinical trials for ITP, 2 cases of acute onset hemoglobinuria consistent with intravascular hemolysis were noted. ${ }^{32}$ Additional cases of acute hemoglobinemia and hemoglobinuria have subsequently been reported to the FDA. ${ }^{33}$ In April of 1998, the manufacturers of anti-D revised the package insert to include reports of hemoglobinuria that was occasionally accompanied by reversible, acute renal impairment. ${ }^{8}$

Gaines reviewed 11 patient reports of hemoglobinuria and noted that 7 of these patients developed significant anemia that required packed red blood cell transfusion orders. ${ }^{33}$ Eight patients experienced new onset or an exacerbation of preexisting renal insufficiency and 2 patients required dialysis. One patient died from pulmonary edema and respiratory distress associated with exacerbated anemia. The authors conjectured several possible mechanisms, including concomitant disorders associated with immune-mediated hemolysis such as undiagnosed Evan's syndrome; Rh phenotype with high expression of D antigen; splenic saturation and resulting diminished capacity for clearance of anti-D-sensitized RBCs; the formation of complement fixing immune complexes in vivo involving anti-D IgG1 or IgG3 aggregates with antiidiotype antibodies in anti-D immunoglobulin that bind to the red cell initiating complement-mediated hemolysis; or improper storage and reconstitution techniques of anti-D. ${ }^{33}$

Gaines subsequently published a review of disseminated intravascular coagulation (DIC) reported to the FDA following anti-D administration. ${ }^{34}$ This case series included 6 patients, 1 child and 5 adults, all of whom died. Gaines conjectured the DIC was causally related to hemoglobinemia and perhaps associated with the passive acquisition of blood group antibodies in anti-D 
immunoglobulin preparation, including high-titer anti-D, low-titer anti-A, anti-B, anti-C, anti-E, anti-Duffy, and anti-Kidd antibodies. ${ }^{34}$

In a letter to the editor, Tarantino et al proposed a "2-hit" phenomenon responsible for the intravascular hemolysis, DIC, and the renal failure sometimes seen after anti-D administration. ${ }^{35}$ Conditions that could have contributed to these complications with anti-D treatment include coexisting autoimmune hemolytic anemia (Evan's syndrome), antiphospholipid antibodies, pre-existing renal insufficiency, and high cytokine induced hemostatic activation. ${ }^{35}$ The authors of the letter recommended examining for hematuria and hemoglobinuria 48 hours after anti-D infusion, screening for and avoiding use of the drug in patients with evidence of possible underlying hemolysis as suggested by an elevated reticulocyte count, and in patients at high risk for hemolysis such as those with positive direct red blood cell antibody test results. ${ }^{35}$

\section{Summary}

Anti-D immunoglobulin therapy has demonstrated efficacy in rapidly increasing the platelet counts in nearly $80 \%$ of children and $70 \%$ of adults with ITP. It appears to be effective only in patients with an intact spleen. However, anecdotal reports of efficacious use in splenectomized patients suggest the there may be some utility in selected patients. A more systematic study of its use in splenectomized patients may be indicated. While a higher dose of $75 \mu \mathrm{g} / \mathrm{kg}$ has shown in one study to result in a more rapid increase in the platelet count, it is uncertain whether this increase in the rate of response is of significant clinical benefit. Anti-D therapy appears to have significant advantages over the use of intravenous immunoglobulin in regards to expense and a longer duration of response. ${ }^{17}$ While intermittent use of anti-D can be used to delay time to splenectomy in patients with chronic ITP, it does not appear to change the natural history of this disorder. It can allow for time for a small number of patients $(15 \%)$ developing a spontaneous remission prior to splenectomy. However, it is uncertain whether use of the newer thrombopoietin receptor agonists could accomplish the same delay in splenectomy with less toxicity. ${ }^{6}$ Finally, while severe and life-threatening toxicities are rare with anti-D therapy, recent recommended guidelines for its use can reduce the risk of these serious events. ${ }^{35}$ Recombinant monoclonal and polyclonal anti-D antibodies have begun to be tested for autologous red cell clearance and a determination of their utility in the management of ITP awaits the results of further clinical studies. ${ }^{36-37}$

\section{Disclosures}

The authors have no conflicts of interest to disclose.

\section{References}

1. Cines, DB, Blanchette VS. Immune thrombocytopenic purpura. $N$ Engl J Med. 2002;346:995-1008.

2. Tarantino MD, Bolton-Maggs PHB. Update on the management of immune thrombocytopenic purpura in children. Curr Opin Hematol. 2007; 14:526-534.

3. Liebman HA. The other thrombocytopenias. Semin Hematol. 2007; 44 (suppl 5):S24-S34.

4. Cines DB, McMillan R. Pathogenesis of chronic immune thrombocytopenic purpura. Curr Opin Hematol. 2007;14:511-514.

5. Godeau B, Provan D, Bussel J. Immune thrombocytopenic purpura in adults. Curr Opin Hematol. 2007;14:535-556.

6. Kuter D, Bussel JB, Lyons RM, et al. Randomized, controlled, 6-month evaluation of AMG-531 in patients with chronic immune thrombocytopenic purpura. Lancet. 2008;371:395-403.

7. Bowman JM. Friesen AD. Pollock JM. Taylor WE. WinRho: Rh immune globulin prepared by ion exchange for intravenous use. Can Med Assoc J. 1980;123:1121-1127.

8. Rho (D) immune globulin intravenous (human): WinRho SDF [package insert]. Winnipeg, Manitoba, Canada: Cangene Corporation; April 1998.

9. Behrens O, Lelle RJ. Rhesus prophylaxis: history and current status. Zentralblatt Gynakol. 1997;119:204-210.

10. Salama A, Kiefel V, Amberg R, Mueller-Eckhart C. Treatment of autoimmune thrombocytopenic purpura with rheus antibodies (antiRho(d). Blut. 1984;49:29-35.

11. Panzer S, Grumayer ER, Hass OA, Niessner H, Graninger W. Efficacy of rhesus antibodies (anti-Rho(D)) in autoimmune thrombocytopenia: Correlation with response to high dose $\mathrm{IgG}$ and the degree of haemolysis. Blut. 1986;52:117-121.

12. Becker T, Kuenzlen E, Salama A, et al. Treatment of childhood idiopathic thrombocytopenic purpura with Rhesus antibodies (antiD). Eur J Pediatr. 1986;145:166-169.

13. Bussel JB, Graziano JN, Kimberly RP, Pahwa S, Aledort LM. Intravenous anti-D treatment of Immune thrombocytopenic purpura: Analysis of efficacy, toxicity and mechanism of effect. Blood. 1991;77:1884-1893.

14. Andrew M, Blanchette VS, Adams M, et al. A multicenter study of the treatment of childhood chronic idiopathic thrombocytopenic purpura with anti-D. J Pediatr. 1992;120:522-527.

15. Blanchette V, Imbach P, Andrew M, et al. Randomized trial of intravenous immunoglobulin $G$, intravenous anti-D and oral prednisone in childhood acute immune thrombocytopenic purpura. Lancet. 1994;334:703-707.

16. Borgna-Pignatti C, Battisti L, Zecca M, Locatelli F. Treatment of chronic childhood immune thrombocytopenic purpura with intramuscular anti-D immunoglobulins. Br J Haematol. 1994;88:618-620.

17. Scaradavou A, Woo B, Woloski BMR, et al. Intravenous anti-D treatment of immune thrombocytopenic purpura: experience in 272 patients. Blood. 1997;89:2689-2700.

18. McMillan R, Wang L, Tomer A, Nichol J, Pistillo J. Suppression of in vitro megakaryocyte production by antiplatelet autoantibodies from adult patients with chronic ITP. Blood. 2004;103:1364-1369.

19. Salama A, Kiefel V, Mueller-Eckhart C. Effect of IgG anti-Rho(D) in adult patients with chronic autoimmune thrombocytopenia. Am J Hematol. 1986;22:241-250.

20. Rossi E, Vimercati AR, Damasio EE, et al. Treatment of idiopathic thrombocytopenic purpura in HIV-positive patients with rhesus antibodies (anti-D). Haematologica. 1987;72:529-32.

21. Boughton BJ, Chakraverty R, Baglin TP, et al. The treatment of chronic idiopathic thrombocytopenia with anti-D (Rho) immunoglobulin: its effectiveness, safety and mechanism of action. Clin Lab Haematol. 1988;10:275-284. 
22. Oksenhendler E, Bierling P, Brossard Y, Schenmetzler C, Girard PM, Seligmann M. Anti-Rh immunoglobulin therapy for human immunodeficiency virus-related immune thrombocytopenic purpura. Blood. 1988;71:1499-1502.

23. Gringeri A, Cattaneo M, Santagostino E, Mannucci PM. Itramuscular anti-D immunoglobulins for home treatment of chronic immune thrombocytopenic purpura. Br J Haematol. 1992;80:337-340.

24. Rodeghiero F, Schiavotto C, Castaman G, Vespignani M, Ruggeri M, Dini E. A follow-up study of 49 adult patients with idiopathic thrombocytopenic purpura treated with high-dose immunoglobulins and anti-D immunoglobulins. Haematologica. 1992;77:248-252.

25. Newman GC, Novoa MV, Fodero EM, et al. A dose of $75 \mathrm{mcg} / \mathrm{kg} / \mathrm{d}$ of IV anti-D increases the platelet count more rapidly and for a longer period of time than $50 \mathrm{mcg} / \mathrm{kg} / \mathrm{d}$ in adults with immune thrombocytopenic purpura. Br J Haematol. 2001;112:1076-1078.

26. Cooper N, Woloski BMR, Fodero EM, et al. Does treatment with intermittent infusions of intravenous anti-D allow a proportion of adults with recently diagnosed immune thrombocytopenic purpura to avoid splenectomy? Blood. 2002;99:1922-1927.

27. George JN, Raskob GE, Vesely SK, et al. Initial management of immune thrombocytopenic purpura in adults: a randomized controlled trial comparing intermittent anti-D with routine care. Am J Hematol. 2002;74:161-169.

28. Coopamah MD, Freedman J, Semple JW. Anti-D initially stimulates an Fc-dependent leukocyte oxidative burst and subsequently suppresses erythrophagocytosis via interleukin-a receptor antagonist. Blood. 2003;102:2862-2867.

29. Crow AR, Lazarus AH. The mechanisms of action of intravenous immunoglobulin and polyclonal anti-D immunoglobulin in the amelioration of immune thrombocytopenic purpura: what do we really know? Transfusion Med Rev. 2008;22:103-116.
30. Cooper N, Heddle NM, de Haas M, et al. Intravenous (IV) anti-D and IV immunoglobulin achieve acute platelet increases by different mechanisms: modulation of cytokine and platelet responses to

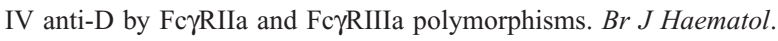
2004;124:511-518.

31. Siragam V, Crow AR, Brinc D, Song S, Freedman J, Lazarus AH. Intravenous immunoglobulin ameliorates ITP via activating $\mathrm{Fc}$ gamma receptors on dendritic cells. Nat Med. 2006;12:600-692.

32. Hong F, Ruiz R, Price H, Griffiths A, Malinoski F, Woloski M. Safety profile of WinRho anti-D. Semin Hematol. 1998;35(suppl 1):9-13.

33. Gaines AR. Acute onset hemoglobinemia and/or hemoglobinuria and sequelae following $\mathrm{Rh}(\mathrm{D})$ immune globulin intravenous administration in immune thrombocytopenic purpura patients. Blood. 2000;95:2523-2529.

34. Gaines AR. Disseminated intravascular coagulation associated with acute hemoglobinemia or hemoglobinuria following $\mathrm{Rh}(\mathrm{D})$ immune globulin intravenous administration for immune thrombocytopenic purpura. Blood. 2005;106:1532-1537.

35. Tarantino MD, Bussel JB, Douglas DB, et al. A closer look at intravascular hemolysis (IVH) following intravenous anti-D for immune thrombocytopenic purpura (ITP). Blood. 2007;109:12.

36. Kumpel BM. Efficacy of RhD monoclonal antibodies in clinical trials as replacement therapy for prophylactic anti-D immunoglobulin: more questions than answers. Vox Sanguinis. 2007;93:99-111.

37. Beliard R, Waegemans T, Notelet D, et al. A human anti-D monoclonal antibody selected for enhanced Fc $\gamma$ RIII engagement clears RhD + autologous red cells in human volunteers as efficiently as polyclonal anti-D antibodies. Br J Haematol. 2008;141:109-119. 